\title{
SUBSTRATO PARA PRODUÇÃO DE MUDAS DE MACACAÚBA (Platymiscium ulei Harms) NO MUNICÍPIO DE AUTAZES, AM
}

\author{
SUBSTRATE FOR PRODUCTION OF MACACAÚBA (Platymiscium ulei Harms) SEEDLINGS IN \\ THE AUTAZES TOWN, AMAZONAS STATE
}

\author{
José Furtado de Miranda ${ }^{1}$ Iza Maria Paiva Batista ${ }^{2}$ Carlos Alberto Franco Tucci ${ }^{3}$ \\ Narrubia Oliveira de Almeida ${ }^{4}$ Marcelo de Almeida Guimarães ${ }^{5}$
}

\section{RESUMO}

A produção de mudas de espécies nativas com qualidade, a fim de se obter sucesso nos reflorestamentos, está muitas vezes relacionada com o nível de eficiência dos substratos utilizados, o objetivo deste trabalho foi estudar diferentes composições de substratos para produção de mudas no município de Autazes, AM. Foram testados oito tratamentos dispostos em delineamento experimental de blocos casualizados, com cinco repetições. Os tratamentos foram: esterco bovino, calagem, fosfatagem corretiva, adubação com NPK, calagem + fosfatagem corretiva, fosfatagem corretiva + adubação com NPK, calagem + adubação com NPK e calagem + fosfatagem corretiva + adubação com NPK. Após 120 dias, foram avaliadas as seguintes características: altura da parte aérea, diâmetro do colo, matéria seca das partes: aérea, raiz e total, e os teores e conteúdos de nutrientes na matéria seca da parte aérea. Os resultados permitem afirmar que todos os tratamentos proporcionaram progressos nos atributos do substrato. Essas melhorias ocasionaram maior disponibilidade de nutrientes para as plantas, os quais proporcionaram um crescimento adequado das mudas de macacaúba. Sendo que as plantas submetidas ao tratamento esterco bovino apresentaram maior absorção de nutriente e maiores taxas de crescimento em menor período de tempo. Sugerindo que este substrato é o mais indicado para a produção de mudas de macacaúba, por promover às mudas maiores taxa de crescimento e em um menor período de tempo.

Palavras-chave: adubação; solo; nutrição de mudas.

\section{ABSTRACT}

The production of native species seedlings with quality in order to achieve success in reforestation is related with the level of efficiency of used substrates. The objective of the study was to assess different substrate compositions for the production of seedlings in the city of Autazes, in Amazonas state. Eight treatments arranged in random blocks experimental design, with five replicates were tested. The treatments were: cattle manure, lime, corrective phosphate, fertilizer with NPK, lime + correction phosphate, corrective phosphate + fertilizer with NPK, lime + fertilization with NPK and lime + corrective phosphate + fertilizer with NPK. After 120 days, the following characteristics were assessed: height of shoot, diameter of the neck, shoot,

1 Licenciado em Ciências Agrárias, Msc., Professor do Colegiado de Ciências Agrárias e do Ambiente, Instituto de Natureza e Cultura, Universidade Federal do Amazonas, Rua 1 de Maio Colônia, CEP 69630-000, Benjamin Constant (AM), Brasil. mirandaufam@hotmail.com

2 Licenciada em Ciências Agrárias, Doutoranda em Agronomia Tropical, Universidade Federal do Amazonas, Av. General Rodrigo Octávio Jordão Ramos, 3000, Campus Universitário, Coroado I, CEP 69077-000, Manaus (AM), Brasil.im_paiva@hotmail

3 Engenheiro Agrônomo, Dr., Professor do Departamento de Engenharia Agrícola e Solos, Faculdade de Ciências Agrárias, Universidade Federal do Amazonas, Av. General Rodrigo Octávio Jordão Ramos, 3000, Campus Universitário, Coroado I, CEP 69077-000, Manaus (AM), Brasil. ctucci@ufam.edu.br

4 Engenheira Florestal, Dra., Professora do Departamento de Ciências Florestais, Faculdade de Ciências Agrárias, Universidade Federal do Amazonas, Av. General Rodrigo Octávio Jordão Ramos, 3000, Campus Universitário, Coroado I, CEP 69077-000, Manaus (AM), Brasil.nalmeida@ufam.edu.br

5 Engenheiro Agrônomo, Dr., Professor do Departamento de Fitotecnia, Centro de Ciências Agrárias, Universidade Federal do Ceará, Av. Mister Hull, 2977, Bloco 805, Campus do Pici, CEP 60021-970, Fortaleza (CE), Brasil. mguimara@hotmail.com

Recebido para publicação em 12/05/2011 e aceito em 21/08/2012 
root and total dry matter, nutrient levels and amounts in shoot dry matter. The results corroborate that all treatments provided improvements in the soil attributes. Those improvements caused larger readiness of nutrients for the plants, which provided an appropriate growth of Macacaúba seedlings. The plants that grew on cattle manure treatment showed higher nutrient absorption and growth rates in a smaller period of time. It suggests that cattle manure soil is the most suitable in the production of Macacaúba seedlings.

Keywords: manuring; soil; seedling nutrition.

\section{INTRODUÇÃO}

A Platymiscium ulei Harms, pertencente à família Fabaceae, conhecida vulgarmente por macacaúba, ocorre em toda região amazônica, sendo mais frequente na região do baixo Amazonas (LOUREIRO et al., 1979). A importância da exploração da macacaúba deve-se a seu alto valor econômico nos mercados nacionais e internacionais de madeiras, sendo uma espécie madeireira promissora no Brasil. Com isso, as informações silviculturais são de grande interesse, já que ela pode se tornar uma espécie a ser utilizada para plantios florestais (SOUZA et al., 2001).

Para macacaúba, a necessidade de definição de técnicas para exploração começa na formação de mudas, principalmente devido ao pouco conhecimento dessa espécie em relação ao manejo nutricional para sua formação. Além disso, para que se produzam mudas de qualidade é necessário o conhecimento das características das espécies, notadamente seus requerimentos nutricionais e suas respostas à correção da acidez do substrato e à adição de fertilizantes (TUCCI, 2005).

Entre os fatores que influenciam a produção de mudas de espécies florestais, destaca-se o substrato que pode ser determinante para o desenvolvimento satisfatório das mudas. A terra de subsolo tem sido muitas vezes usada como base de substrato, pois é isento de sementes de plantas indesejáveis e micro-organismos patogênicos. Todavia, o material de subsolo, em geral, é ácido e contém níveis baixos de nutrientes, tornando-se necessária a sua correção para que possa ser utilizado.

A acidez do solo e a deficiência de nutrientes podem ser corrigidas facilmente por meio da calagem e fertilização mineral (PAIVA e GOMES, 2000). Outra opção é a utilização de adubos orgânicos, que, dependendo das quantidades aplicadas, proporcionam aumento no teor de matéria orgânica, com consequente aumento na capacidade de troca de cátions e melhora da estrutura do solo (ARTUR et al., 2007). A matéria orgânica tem efeito significativo para o desenvolvimento dos vegetais. Possui estreita relação com a capacidade do solo em fornecer nutrientes às plantas, em que processos de mineralização e imobilização orgânica, e de dessorção e adsorção de cátions e ânions, entre outros, governam a disponibilidade dos nutrientes às mesmas (RAIJ, 1991). Entre os adubos orgânicos, o esterco bovino é o mais usado e tem levado a bons resultados na produção de mudas de espécies florestais.

Devido à grande quantidade e facilidade de obtenção no município de Autazes, consequência da grande quantidade de animais existentes, o esterco bovino pode ser uma alternativa economicamente viável para a produção de mudas de espécies florestais. Prova disso é que o solo próximo aos estábulos tem se tornado cada vez mais fértil, devido principalmente ao escorrimento superficial do esterco, que, ao atingir solos mais aerados, infiltra e os fertiliza. Fato contrário a esse é a dificuldade que agricultores têm em adquirir corretivos e fertilizantes. Isso ocorre principalmente devido às baixas condições econômicas dos produtores e à falta de uma política direcionada para o desenvolvimento do setor primário, tornando cada vez mais difícil o avanço do mesmo.

Em face da inexistência de estudos enfocando o melhor substrato para produzir mudas da espécie macacaúba, o objetivo deste trabalho foi estudar diferentes composições de substratos para produção de mudas no município de Autazes, AM.

\section{MATERIAL E MÉTODO}

O experimento foi conduzido no viveiro de mudas da Prefeitura Municipal de Autazes, no período de agosto de 2004 a agosto de 2005.

As sementes de macacaúba foram coletadas em outubro de 2004, em um total de duas (02) matrizes localizadas nas várzeas da margem esquerda do Rio Autaz-açu no Município de Autazes-AM. As sementes foram selecionadas segundo Lamprecht (1990).

A semeadura foi realizada em sementeira, utilizando-se como substrato areia lavada com hipoclorito de sódio. Após o aparecimento de dois pa- 
res de folhas, as plântulas foram selecionadas, para homogeneização quanto ao tamanho e, em seguida, foram repicadas para os sacos plásticos com capacidade de $4,5 \mathrm{~kg}$ de substrato que continham distintos tratamentos.

Para a produção das mudas, o solo utilizado como substrato foi coletado na camada subsuperficial (20 a $40 \mathrm{~cm}$ de profundidade) de um Latossolo Amarelo, textura média, coletado no município de Autazes, cujas coordenadas em UTM são 21M0263344 e 9603224. O material foi seco ao ar, passado em peneira de $4 \mathrm{~mm}$ de abertura e subamostras do solo foram passadas em peneiras de $2 \mathrm{~mm}$, sendo submetidas a análises químicas de acordo com metodologia proposta pela Embrapa (1999), sendo apresentadas na Tabela 1.

Os tratamentos foram dispostos em blocos casualizados, com oito tratamentos e cinco repetições e cada parcela composta por cinco plantas, sendo: Tratamento 1 (T1) esterco bovino, Tratamento 2 (T2) calagem, Tratamento 3 (T3) fosfatagem corretiva, Tratamento 4 (T4) adubação com NPK, Tratamento 5 (T5) calagem + fosfatagem corretiva, Tratamento 6 (T6) fosfatagem corretiva + adubação com NPK, Tratamento 7 (T7) calagem + adubação com NPK e Tratamento 8 (T8) calagem + fosfatagem corretiva + adubação com NPK.

As quantidades fornecidas dos adubos foram variáveis de acordo com cada fonte. De modo que, o esterco bovino correspondeu à proporção 2:1 em peso, sendo uma parte de esterco para duas partes de solo, conforme sugestões de Carvalho Filho et al. (2004). O esterco utilizado foi curtido, seco ao ar e homogeneizado para ser misturado ao Latossolo de forma uniforme.

A calagem correspondeu a 2,0 t.ha-1, o equivalente $1 \mathrm{~kg} \cdot \mathrm{m}^{-3}$ de solo, segundo método proposto por Catani e Alonso (1969) e sugerida por Tucci (1999) como adequada para os solos da região da Amazônia. A calagem foi realizada com trinta (30) dias de antecedência para que ocorresse a solubilização e reação do calcário com o solo, utilizando uma mistura de carbonato de cálcio e carbonato de magnésio (P.A.), na proporção 4:1 em peso, respectivamente.
A fosfatagem corretiva foi realizada com dose equivalente a $10 \mathrm{~kg}$ de $\mathrm{P}_{2} \mathrm{O}_{5}$ por $\mathrm{m}^{-3}$ de solo, de acordo com sugestões de Tucci et al. (2007), utilizando como fonte o superfosfato triplo.

A adubação com NPK correspondeu na adição de 100-300-200 kg.ha-1 de $\mathrm{N}^{-} \mathrm{P}_{2} \mathrm{O}_{5}-\mathrm{K}_{2} \mathrm{O}$, o equivalente a 50-150-100 g.m $\mathrm{m}^{-3}$ de solo, cuja recomendação foi adaptada de Tucci et al. (2002). Como fonte de N- $\mathrm{P}_{2} \mathrm{O}_{5}-\mathrm{K}_{2} \mathrm{O}$ foi utilizada ureia, superfosfato triplo e cloreto de potássio, respectivamente. Tanto a fosfatagem corretiva e a adubação com NPK foram aplicados diretamente no solo na forma de granulado.

Com 30 dias após a incubação, subamostras do solo foram retiradas para novas análises químicas (resultados expressos na Tabela 2), sendo no mesmo período e após, a cada 15 dias, até o final do experimento, adicionada adubação com micronutrientes em todas as parcelas, excetuando-se as parcelas que continham o esterco de gado. Isto correspondeu à aplicação de $89 \mathrm{mg}$ de $\mathrm{B}$ por $\mathrm{m}^{-3} ; 111 \mathrm{mg}$ de $\mathrm{Cu}$ por $\mathrm{m}^{-3} ; 556 \mathrm{mg}$ de $\mathrm{Mn}$ por $\mathrm{m}^{-3} ; 556 \mathrm{mg}$ de $\mathrm{Zn}$ por $\mathrm{m}^{-3} ; 11 \mathrm{mg}$ de Mo por $\mathrm{m}^{-3}$ e $889 \mathrm{mg}$ de Fe por $\mathrm{m}^{-3}$. As doses foram adaptadas a partir das sugestões de Tucci et al. (2007).

Aos 120 dias após o transplantio, foram avaliados: a altura da parte aérea $(\mathrm{cm})$, tomando-se como referência a distância do nível do solo ao ápice, e o diâmetro do colo ( $\mathrm{mm}$ ) tomado na região do coleto da planta.

Para análise de biomassa, as plantas foram cortadas na altura do colo separando-se a parte aérea do sistema radicular. Após, foi realizada a lavagem das raízes em água corrente para retirada do excesso de substrato, para isso utilizou-se peneira para evitar a perda de material vegetal. Depois de lavado, o material vegetal foi separado de acordo com o tratamento, sendo, então, acondicionado em saco de papel e levado para secagem, até atingir massa constante, em estufa com temperatura regulada a $65^{\circ} \mathrm{C}$. Após, foi determinada a matéria seca da parte aérea (MSPA, g), raiz (MSR, g) e total (MST, g). Posteriormente, os materiais vegetais obtidos em cada um dos tratamentos foram submetidos a análises químicas para a determinação dos teores de $\mathrm{N}$,

TABELA 1: Análise do solo utilizado como substrato.

TABLE 1: Analysis of soil used as substrate.

\begin{tabular}{|c|c|c|c|c|c|c|c|c|c|c|c|c|c|}
\hline $\mathrm{MO}$ & $\mathrm{ph}$ & $\mathrm{Al}^{3+}$ & $\mathrm{Ca}^{2+}$ & $\mathrm{Mg}^{2+}$ & $\mathrm{H}+\mathrm{Al}$ & $\mathrm{P}$ & $\mathrm{K}$ & S & $\mathrm{t}$ & $\mathrm{T}$ & $\mathrm{V}$ & $\mathrm{m}$ & argila \\
\hline $\mathrm{g} / \mathrm{kg}^{-1}$ & $\left(\mathrm{H}_{2} \mathrm{O}\right)$ & ----- & $\begin{array}{l}---c \\
-c\end{array}$ & $\mathrm{c}_{\mathrm{c}} \mathrm{kg}$ & ------- & & & & $\mathrm{nol}_{\mathrm{c}} /$ & - & & $--(\%$ & ----- \\
\hline 25,1 & 3,8 & 3,7 & 0,5 & 0,05 & 9,81 & 5 & 24 & 0,6 & 4,3 & 10,4 & 6 & 86 & 26 \\
\hline
\end{tabular}


$\mathrm{P}, \mathrm{K}, \mathrm{Ca}, \mathrm{Mg}, \mathrm{Fe}, \mathrm{Cu}, \mathrm{Zn}$ e $\mathrm{Mn}$ da parte aérea, sendo utilizado para isso o procedimento descrito por Malavolta et al. (1997). A estimativa dos conteúdos totais de $\mathrm{N}, \mathrm{P}, \mathrm{K}, \mathrm{Ca}, \mathrm{Mg}, \mathrm{Fe}, \mathrm{Cu}, \mathrm{Zn}$ e $\mathrm{Mn}$ da parte aérea foram obtidas multiplicando-se o teor de cada elemento pela matéria seca correspondente.

Os dados obtidos foram submetidos à análise de variância utilizando o programa SAEG e as médias foram comparadas pelo teste de Scott-Knott ao nível de $5 \%$ de probabilidade.

\section{RESULTADOS E DISCUSSÃO}

As aplicações de esterco, calcário, fertilizantes e suas combinações, promoveram alterações na composição química do substrato em diferentes magnitudes (Tabela 2).

De modo geral, o esterco bovino influenciou positivamente todos os atributos de fertilidade do substrato. Observa-se que a aplicação do esterco proporcionou redução no valor da acidez, bem como dos demais componentes relacionados a este (Tabela 2), culminando assim, em maior disponibilidade de nutrientes para as plantas. Essa afirmação é confirmada ao observarmos elevação nos teores

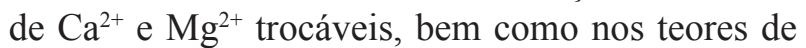
$\mathrm{K}$ e $\mathrm{P}$ disponíveis (Tabela 2) no substrato, 30 dias após o tratamento. Essas observações concordam com as realizadas por Yagi et al. (2003), Caetano e Carvalho (2006) e Artur et al. (2007), que observaram efeito positivo e significativo para todos os atributos do substrato após serem tratados com esterco.

Comparando-se o tratamento esterco bovino com os demais tratamentos que utilizaram cal
$\left(\mathrm{CaCO}_{3}\right)$, verificou-se semelhança nos valores dos parâmetros $\mathrm{pH}, \mathrm{Al}^{3+}$, acidez potencial $(\mathrm{H}+\mathrm{AL})$ e saturação por bases $(\mathrm{V})$. Isto sugere que o $\mathrm{CaCO}_{3}$ pode ser substituído por esterco bovino para promover a redução nos componentes de acidez do solo.

O substrato tratado com fosfatagem corretiva apresentou aumento dos níveis de $\mathrm{Ca}^{2+}$ e também elevação do $\mathrm{pH}$ (Tabela 2). $\mathrm{O}$ aumento dos teores de $\mathrm{Ca}^{2+}$ deveu-se principalmente à presença deste nutriente na fonte do fertilizante utilizado. Já a elevação do $\mathrm{pH}$, causada pela fosfatagem, pode ter ocorrido devido à adsorção específica de fósforo no solo, que, por troca de ligantes (GUARÇONI e MENDONÇA, 2003 e PAIM et al., 2006), libera para a solução do solo grupamentos hidroxila $\left(\mathrm{OH}^{-}\right)$, promovendo assim, o aumentando o $\mathrm{pH}$ do substrato.

De acordo com os conteúdos de macronutrientes encontrados na matéria seca da parte aérea, observa-se (Tabela 3) que a sequência de exigência nutricional das mudas de macacaúba, considerando a quantidade absorvida pelas mudas, segue a seguinte ordem: $\mathrm{N}>\mathrm{K}>\mathrm{Ca}>\mathrm{P}>\mathrm{Mg}>\mathrm{S}$.

Pode-se observar na Tabela 3 que o substrato tratado com esterco bovino apresentou os maiores conteúdos de N, P, K, Ca, Mg e S na parte aérea das mudas, diferindo estatisticamente dos demais substratos submetidos a diferentes tratamentos. Provavelmente, o maior acúmulo observado para o tratamento esterco de curral, deveu-se a melhorias nas condições do solo, sobretudo da fertilidade pelo fornecimento adicional de nutrientes através da incorporação do esterco bovino. Estes resultados concordam com os apresentados por Artur et al.

TABELA 2: Características químicas do solo das parcelas experimentais em função dos tratamentos, antes do transplantio.

TABLE 2: Soil chemical characteristics of experimental plots on the basis of treatments before transplanting.

\begin{tabular}{cccccccccccc}
\hline \multirow{2}{*}{ Tratamento } & $\mathrm{pH}$ & $\mathrm{Al}^{3+}$ & $\mathrm{H}+\mathrm{Al}$ & $\mathrm{Ca}^{2+}$ & $\mathrm{Mg}^{2+}$ & $\mathrm{K}$ & $\mathrm{P}$ & $\mathrm{t}$ & $\mathrm{T}$ & $\mathrm{V}$ & $\mathrm{M}$ \\
\cline { 2 - 11 } & $\left(\mathrm{H}_{2} \mathrm{O}\right)$ & $-----------\mathrm{cmol}_{\mathrm{c}} / \mathrm{kg}------------$ & $--\mathrm{mg} / \mathrm{kg}--$ & \multicolumn{1}{c}{$\mathrm{cmol}_{\mathrm{c}} / \mathrm{kg}$} & $--(\%)---$ \\
\hline (T1) Esterco bovino & 5,7 & 0,09 & 6,31 & 2,65 & 2,19 & 210 & 22 & 5,46 & 11,68 & 46 & 1,6 \\
(T2) Cal & 5,6 & 0,09 & 6,10 & 2,54 & 0,14 & 23 & 148 & 3,66 & 8,83 & 31 & 25 \\
(T3) FC & 5,4 & 0,08 & 7,72 & 3,01 & 0,26 & 40 & 241 & 4,20 & 10,80 & 31 & 20 \\
(T4) NPK & 5,6 & 1,54 & 6,06 & 1,19 & 0,24 & 40 & 12 & 3,06 & 7,59 & 20 & 50 \\
(T5) Cal+FC & 5,4 & 0,78 & 5,56 & 2,10 & 0,50 & 28 & 128 & 3,45 & 8,23 & 32 & 22 \\
(T6) FC+NPK & 5,4 & 0,59 & 7,34 & 3,25 & 0,39 & 29 & 267 & 4,30 & 11,05 & 34 & 14 \\
(T7) Cal+NPK & 5,5 & 0,54 & 6,68 & 3,00 & 0,41 & 30 & 220 & 4,02 & 10,17 & 34 & 13 \\
(T8) Cal+FC+NPK & 5,5 & 1,05 & 6,10 & 2,15 & 0,24 & 31 & 135 & 3,51 & 8,57 & 29 & 30 \\
\hline
\end{tabular}

Em que: $\mathrm{FC}=$ Fosfatagem corretiva; $\mathrm{Cal}=$ Calagem; $\mathrm{NPK}=\mathrm{N}-\mathrm{P}_{2} \mathrm{O}_{5}-\mathrm{K}_{2} \mathrm{O}$. 
TABELA 3: Conteúdo dos macronutrientes na matéria seca da parte aérea em mudas de macacaúba, avaliadas 120 dias após transplantio em função dos tratamentos.

TABLE 3: Content of nutrients in shoot dry matter in seedlings of Macacaúba evaluated 120 days after transplanting depending on the treatments.

\begin{tabular}{|c|c|c|c|c|c|c|}
\hline \multirow{2}{*}{ Tratamento } & $\mathrm{N}$ & $\mathrm{P}$ & K & $\mathrm{Ca}$ & $\mathrm{Mg}$ & $\mathrm{S}$ \\
\hline & \multicolumn{6}{|c|}{ 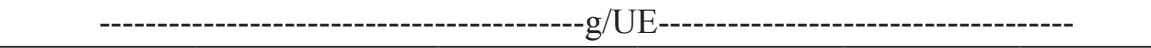 } \\
\hline (T1) Esterco bovino & $122,64 \mathrm{a}$ & $15,60 \mathrm{a}$ & $107,83 \mathrm{a}$ & $39,82 \mathrm{a}$ & $15,16 \mathrm{a}$ & 8,50 a \\
\hline (T2) $\mathrm{Cal}$ & $8,97 \mathrm{~b}$ & $1,08 \mathrm{~b}$ & $7,63 \mathrm{~b}$ & $5,66 \mathrm{c}$ & $1,85 \mathrm{c}$ & $0,94 \mathrm{c}$ \\
\hline (T3) FC & $19,14 \mathrm{~b}$ & $3,99 \mathrm{~b}$ & $16,71 \mathrm{~b}$ & $16,79 \mathrm{~b}$ & $3,53 \mathrm{~b}$ & $2,43 \mathrm{~b}$ \\
\hline (T4) NPK & $16,59 \mathrm{~b}$ & $1,80 \mathrm{~b}$ & $21,00 \mathrm{~b}$ & $5,39 \mathrm{c}$ & $1,80 \mathrm{c}$ & $1,23 \mathrm{c}$ \\
\hline (T5) $\mathrm{Cal}+\mathrm{FC}$ & $22,39 \mathrm{~b}$ & $3,16 \mathrm{~b}$ & $14,71 \mathrm{~b}$ & $12,09 \mathrm{~b}$ & $3,74 \mathrm{~b}$ & $1,71 \mathrm{~b}$ \\
\hline (T6) $\mathrm{FC}+\mathrm{NPK}$ & $21,42 \mathrm{~b}$ & $3,75 \mathrm{~b}$ & $20,99 \mathrm{~b}$ & $17,91 \mathrm{~b}$ & $2,41 \mathrm{c}$ & $2,51 \mathrm{~b}$ \\
\hline (T7) $\mathrm{Cal}+\mathrm{NPK}$ & $26,30 \mathrm{~b}$ & $3,42 \mathrm{~b}$ & $19,71 \mathrm{~b}$ & $13,00 \mathrm{~b}$ & $3,95 \mathrm{~b}$ & $1,93 \mathrm{~b}$ \\
\hline (T8) $\mathrm{Cal}+\mathrm{FC}+\mathrm{NPK}$ & $37,69 \mathrm{~b}$ & $4,57 \mathrm{~b}$ & $19,73 \mathrm{~b}$ & $16,32 \mathrm{~b}$ & $4,08 \mathrm{~b}$ & $2,03 \mathrm{~b}$ \\
\hline
\end{tabular}

Em que: $\mathrm{FC}=$ Fosfatagem corretiva $; \mathrm{Cal}=$ Calagem; $\mathrm{NPK}=\mathrm{N}_{-} \mathrm{P}_{2} \mathrm{O}_{5}-\mathrm{K}_{2} \mathrm{O} ;(\mathrm{g} / \mathrm{UE})=\mathrm{g} /$ unidade experimental. Letras distintas entre linhas demonstram diferenças estatísticas pelo teste de Scott-Knott a $5 \%$ de probabilidade.

(2007) e Yagi et al. (2003), que também verificaram aumento nos teores $\mathrm{P}, \mathrm{K}, \mathrm{Ca}$ e $\mathrm{Mg}$ com a aplicação de esterco e vermicomposto de esterco bovino, respectivamente.

No que se refere aos micronutrientes analisados, verifica-se maiores conteúdos destes no tratamento esterco bovino (Tabela 4). Os resultados permitem inferir que a presença do esterco bovino promoveu maior disponibilidade, na solução do solo, de micronutrientes como $\mathrm{Cu}, \mathrm{Fe}, \mathrm{Mn}$ e $\mathrm{Zn}$, o que certamente contribuiu para maiores acúmulos destes nas mudas analisadas. Este resultado pode estar relacionado aos compostos quelatados (substâncias orgânicas que envolvem os nutrientes impedindo que sejam ligados a outro elemento, o que poderia causar a sua imobilização), fazendo com que cheguem até a rizosfera para serem absorvidos pelas raízes das plantas. De acordo com Silva e Mendonça (2007), a reação de complexação e quelação de metais pela matéria orgânica do solo tem papel importante em vários processos no solo, como: o intemperismo, a disponibilidade de nutrientes e os aspectos ambientais do mesmo. De acordo com Bayer e Mielniczuk (1999) e Miyazawa et al. (2000), a disponibilidade de nutrientes para as culturas, a capacidade de troca de cátions e a complexação do $\mathrm{Al}^{3+}$ e do $\mathrm{Fe}^{2+} / \mathrm{Fe}^{3+}$ são destacadas como características químicas do solo que são influencia-

TABELA 4: Conteúdo dos micronutrientes na matéria seca da parte aérea em mudas de macacaúba, avaliadas 120 dias após transplantio em função dos tratamentos.

TABLE 4: Content of micronutrients in the shoot dry matter in seedlings of Macacaúba evaluated 120 days after transplanting depending on the treatments.

\begin{tabular}{ccccc}
\hline \multirow{2}{*}{ Tratamentos } & $\mathrm{Cu}$ & $\mathrm{Fe}$ & $\mathrm{Mn}$ & $\mathrm{Zn}$ \\
\cline { 2 - 5 } & & -------------------- & $3639 \mathrm{a}$ \\
\hline (T1) Esterco bovino & $625 \mathrm{a}$ & $12268 \mathrm{a}$ & $2239 \mathrm{a}$ & $617 \mathrm{~b}$ \\
(T2) Cal & $41 \mathrm{~b}$ & $2025 \mathrm{~b}$ & $187 \mathrm{~b}$ & $829 \mathrm{~b}$ \\
(T3) FC & $70 \mathrm{~b}$ & $3782 \mathrm{~b}$ & $714 \mathrm{~b}$ & $508 \mathrm{~b}$ \\
(T4) NPK & $85 \mathrm{~b}$ & $3726 \mathrm{~b}$ & $424 \mathrm{~b}$ & $834 \mathrm{~b}$ \\
(T5) Cal+FC & $68 \mathrm{~b}$ & $3552 \mathrm{~b}$ & $387 \mathrm{~b}$ & $1386 \mathrm{~b}$ \\
(T6) FC+NPK & $26 \mathrm{~b}$ & $4897 \mathrm{~b}$ & $508 \mathrm{~b}$ & $578 \mathrm{~b}$ \\
(T7) Cal+NPK & $77 \mathrm{~b}$ & $3854 \mathrm{~b}$ & $452 \mathrm{~b}$ & $1343 \mathrm{~b}$ \\
(T8) Cal+FC+NPK & $110 \mathrm{~b}$ & $5051 \mathrm{~b}$ & $502 \mathrm{~b}$ & $\mathrm{Cx}$ \\
\hline
\end{tabular}

Em que: $\mathrm{FC}=$ Fosfatagem corretiva; $\mathrm{Cal}=$ Calagem; $\mathrm{NPK}=\mathrm{N}-\mathrm{P}_{2} \mathrm{O}_{5}-\mathrm{K}_{2} \mathrm{O} ;(\mathrm{mg} / \mathrm{UE})=\mathrm{mg} /$ unidade experimental. Letras distintas entre linhas demonstram diferenças estatísticas pelo teste de Scott-Knott a $5 \%$ de probabilidade. 
das pela quantidade e tipo de matéria orgânica contida no mesmo.

Os resultados apresentados concordam com os obtidos por Dechen e Nachtigall (2006) e Mobricci (2006), que verificaram elevações nas concentrações de $\mathrm{Cu}$, Fe, Mn e Zn pela aplicação de esterco bovino em solos.

Os resultados de diâmetro e altura observados (Tabela 5) mostram que os tratamentos afetaram de maneira distinta o crescimento em altura e diâmetro das mudas. Observa-se que o substrato adubado com esterco bovino foi significativamente superior, quando comparado aos demais tratamentos. Provavelmente a superioridade em altura e diâmetro das mudas, neste tratamento, esteja relacionada à maior disponibilidade de nutrientes nesse substrato (Tabelas 3 e 4). Além disso, o esterco bovino promove melhores condições físicas e biológicas do solo, contribuindo para a manutenção de sua fertilidade. Cunha et al. (2005), trabalhando com ipê-roxo (Tabebuia impetiginosa Mart. Ex. Dc.), constataram que as mudas submetidas ao substrato com composto orgânico obtiveram os maiores rendimentos.

No entanto, quase todos os tratamentos aplicados atenderam ao padrão de qualidade de mudas apresentado por Gonçalves et al. (2000), que em um ensaio experimental indicou que mudas de boa qualidade deveriam apresentar entre 20 a $35 \mathrm{~cm}$ de altura e 5 a $10 \mathrm{~mm}$ de diâmetro do colo. A exceção é o tratamento esterco bovino, que apresentou em média $61 \mathrm{~cm}$ de altura, ou seja, valor superior ao padrão mencionado anteriormente. Esse resultado sugere a possibilidade de produção de mudas de macacaúba em um menor período de tempo (menos de quatro meses), podendo também ocorrer a utilização de menores proporções de esterco bovino, o que resultaria em menores custos com substrato.

Com relação à biomassa seca da parte aérea, raiz e total (Tabela 5), verifica-se que as mudas de macacaúba conduzidas no tratamento esterco bovino diferenciaram-se significativamente dos demais tratamentos. Esse resultado é consequência dos maiores valores em altura e diâmetro das mudas submetidas a este tratamento. Os resultados apresentados neste trabalho concordam com os obtidos por Carvalho Filho et al. (2002), que testando a influência de diferentes misturas de substrato sobre o rendimento e a biomassa de cassia-grande (Cassia grandis Linn.), observaram que as mudas submetidas ao substrato com esterco bovino obtiveram maior biomassa. De forma semelhante, estudos com as espécies calabura (Muntingia calabura L.) e freijó-louro (Cordia alliodora Ruiz\&Pav.) mostraram efeito positivo no rendimento de mudas, em substrato contendo esterco bovino (CASTRO et al., 1996; VIEIRA et al., 1998).

Por outro lado, o substrato com apenas calagem proporcionou menor valor de biomassa seca, conforme mostra a Tabela 5. Isto sugere que, com base no peso seco, a calagem como prática isolada não proporciona ambiente favorável para o crescimento das mudas de macacaúba, embora tenha apresentado valores de altura e diâmetro dentro dos padrões de qualidade. Entretanto, vale salientar que apesar das mudas terem apresentado rendimento em altura e diâmetro adequados, esse tratamento sem-

TABELA 5: Características de crescimento das plantas de macacaúba, avaliadas 120 dias após transplantio em função dos tratamentos.

TABLE 5: Characteristics of growth of plants Macacaúba evaluated 120 days after transplanting, depending on the treatments.

\begin{tabular}{cccccc}
\hline \multirow{2}{*}{ Tratamento } & Altura & Diâmetro & \multicolumn{3}{c}{ Matéria Seca $(\mathrm{g} / \mathrm{UE})$} \\
\cline { 2 - 6 } & $(\mathrm{cm})$ & $(\mathrm{mm})$ & Parte aérea & Raiz & Total \\
\hline (T1) Esterco bovino & $63,75 \mathrm{a}$ & $9,875 \mathrm{a}$ & $53,96 \mathrm{a}$ & $25,53 \mathrm{a}$ & $79,45 \mathrm{a}$ \\
(T2) Cal & $30,07 \mathrm{~b}$ & $4,875 \mathrm{~b}$ & $6,40 \mathrm{~b}$ & $5,70 \mathrm{~b}$ & $12,10 \mathrm{~b}$ \\
(T3) FC & $39,50 \mathrm{~b}$ & $6,360 \mathrm{~b}$ & $12,62 \mathrm{~b}$ & $12,58 \mathrm{~b}$ & $25,20 \mathrm{~b}$ \\
(T4) NPK & $37,15 \mathrm{~b}$ & $5,915 \mathrm{~b}$ & $10,90 \mathrm{~b}$ & $11,98 \mathrm{~b}$ & $22,88 \mathrm{~b}$ \\
(T5) Cal+FC & $40,60 \mathrm{~b}$ & $6,575 \mathrm{~b}$ & $12,38 \mathrm{~b}$ & $11,40 \mathrm{~b}$ & $23,78 \mathrm{~b}$ \\
(T6) FC+NPK & $33,75 \mathrm{~b}$ & $5,767 \mathrm{~b}$ & $11,58 \mathrm{~b}$ & $9,95 \mathrm{~b}$ & $21,53 \mathrm{~b}$ \\
(T7) Cal+NPK & $43,18 \mathrm{~b}$ & $6,610 \mathrm{~b}$ & $13,80 \mathrm{~b}$ & $13,90 \mathrm{~b}$ & $22,70 \mathrm{~b}$ \\
(T8) Cal+FC+NPK & $44,00 \mathrm{~b}$ & $6,492 \mathrm{~b}$ & $16,15 \mathrm{~b}$ & $14,60 \mathrm{~b}$ & $30,75 \mathrm{~b}$ \\
\hline
\end{tabular}

Em que: $\mathrm{FC}=$ Fosfatagem corretiva; $\mathrm{Cal}=$ Calagem; $\mathrm{NPK}=\mathrm{N}-\mathrm{P}_{2} \mathrm{O}_{5}-\mathrm{K}_{2} \mathrm{O} ;(\mathrm{g} / \mathrm{UE})=\mathrm{g} /$ unidade experimental. Letras distintas entre linhas demonstram diferenças estatísticas pelo teste de Scott-Knott a $5 \%$ de probabilidade. 
pre proporcionou menores valores, o que pode ser explicado pelo fato do substrato apresentar baixo teor de nutrientes no solo (Tabela 1), consequentemente, as mudas não responderam à aplicação somente de calcário. Pelo exposto, o crescimento superior das mudas no substrato com esterco bovino indica a importância deste, para produção de mudas de melhor qualidade (COSTA et al., 2005).

\section{CONCLUSÕES}

O substrato com esterco bovino ocasionou maior disponibilidade e acumulação de nutrientes para as mudas, o que possivelmente proporcionou melhores condições para o crescimento e desenvolvimento destas.

Os tratamentos feitos a base de adubos químicos, apesar de não terem proporcionado maiores valores das variáveis analisadas, atenderam aos padrões de qualidade exigidos.

\section{REFERÊNCIAS BIBLIOGRÁFICAS}

ARTUR, A. G. et al. Esterco bovino e calagem para formação de mudas de guanandi. Pesquisa Agropecuária Brasileira, Brasília, v. 42, n. 6, p. 843-850, 2007.

BAYER, C.; MIELNICZUK, J.. Dinâmica e função da matéria orgânica. In: SANTOS, G.A.; CAMARGO, F. A. O. Fundamentos da matéria orgânica do solo. Gênesis: Rio Grande Sul. 1999, p. 9-26.

CAETANO, L. C. S.; CARVALHO. A. J. C. Efeito da adubação com boro e esterco bovino sobre a produtividade da figueira $\mathrm{e}$ as propriedades químicas do solo. Ciência Rural, Santa Maria, v. 36, n. 4, p. 1150-1155, jul./ago. 2006.

CARVALHO FILHO, J. L. S. et al. Produção de mudas de Cássia grandi L. em diferentes ambientes, recipientes e misturas de substrato. Revista Ceres, Viçosa, v. 49, n. 284, p. 341-352, jul./ago. 2002.

CARVALHO FILHO, J. L. S. et al. Produção de mudas de Angelim (Andira fraxiniflora Benth.) em diferentes ambientes, recipientes e substratos. Revista Ciência Agronômica, Ceará, v. 35, n. 1, p. 61-67, jan./jun. 2004.

CASTRO, E. M. et al. Efeito de substrato na produção de mudas de calabura (Muntingia calabura L.). Ciência e Agrotecnologia, Lavras, v. 20, n. 3, p. 366-370, jun. 1996.

CATANI, R. A.; ALONSO. O. Avaliação da exigência de calcário do solo. Piracicaba: Anais da
Escola Superior de Agricultura Luiz de Queiroz, Piracicaba, n. 26, p. 141-156. 1969.

COMISSÃO DE FERTILIDADE DE SOLOS DO ESTADO DE MINAS GERAIS.

Recomendações para o uso de corretivos e fertilizantes em Minas Gerais: $5^{\text {a }}$ aproximação. Viçosa, 1999. 359 p.

COSTA, M. C. et al. Substrato para produção de mudas de jenipapo (Genipa americana L.). Pesquisa Agropecuária Tropical, Goiânia, v. 35, n. 1, p. 19-24, jan./abr. 2005.

CUNHA, A. O. et al. Efeito de substrato e das dimensões dos recipientes na qualidade de mudas de Tabebuia impetiginosa (Mart. Ex D. C.) standl. Revista Árvore, Viçosa, v. 29, n. 4, p. 507-516, out./dez. 2005.

DECHEN, A. R.; NACHTIGALL. G. R. Micronutrientes. In: Nutrição mineral de plantas. Ed. FERNANDES, M. S., Viçosa: Sociedade Brasileira de Ciências do Solo. 2006. p. 327-354.

EMBRAPA. Manual de análises químicas de solos, plantas e fertilizantes. Brasília. 1999. 370 p. GONÇALVES, J. L. et al. Produção de mudas de espécies nativas: substrato, nutrição, sombreamento e fertilização. In: GONÇALVES, J. L. M. et. al. (Ed.). Nutrição e fertilização florestal. Piracicaba: IPEF. 2000. p.309-350.

GUARÇONI, A.; MENDONÇA. E. S. Capacidade tampão de $\mathrm{pH}$ do solo e disponibilidade de fósforo pela adição de composto orgânico. Magistra, Cruz das Almas, v. 15, n. 2, p. 217-221. jul./dez. 2003.

LAMPRECHT, H. Silvicultura nos Trópicos: ecossistemas florestais e respectivas espécies arbóreas - possibilidades e métodos de aproveitamento sustentado. Eschoborn: Dt. Ges. fur. Techn. Zusammenarbeit (GTZ) GmbH, 1990. $343 \mathrm{p}$.

LOUREIRO, A. A. et al. Essências madeireiras da Amazônia. Manaus: INPA, 1979. 169 p. v. 2.

MALAVOLTA, E. et al. Avaliação do estado nutricional de plantas: Princípios e aplicações. 2. ed. Piracicaba: POTAFOS, 1997. 319 p.

MIYAZAWA, M. et al. Neutralização da acidez do perfil do solo por resíduos vegetais. Informações Agronômicas, Piracicaba, v. 92, n. 1, p. 1-8, jan. 2000.

MOBRICCI, C. A. N. Adubação mineral, esterco de curral e lodo de esgoto no desenvolvimento inicial do cafeeiro. 2006. 64 f. Dissertação (Mestrado em Agronomia) - Universidade Estadual Paulista, Botucatu, 2006. 
PAIM, L. A. et al. Estudos dos efeitos do silício e do fósforo na redução da disponibilidade de metais pesados em área de mineração. Química nova, São Paulo, v. 29, n. 1, p. 28-33, jan./fev. 2006.

PAIVA, H. N.; GOMES. J. M. Viveiros florestais 2. ed. Viçosa: Universidade Federal de Viçosa, 2000. 69 p. (Cadernos didáticos).

RAIJ, B. V. Fertilidade do solo e adubação. São Paulo: Agronômica Ceres, 1991. 343 p.

SILVA, I. R.; MENDONÇA, E. S. Matéria orgânica do solo. In: Fertilidade do solo. Eds. NOVAIS, R. F. et al. Viçosa: Sociedade Brasileira de Ciência do Solo. 2007. p. 275-374.

TUCCI, C. A. F. Seleção de métodos de laboratório para estimativa da necessidade de calagem em alguns solos da Amazônia. Revista da Universidade do Amazonas: Série Ciências Agrárias, Manaus, v. 8, n. 1/2, p. 1-19, 1999.

TUCCI, C. A. F. et al. Adubação e calagem para a formação de mudas de sumaúma (Ceiba pentandra
(L.) Gaerth). Revista da Universidade Amazonas: Série Ciências Agrárias, Manaus, v. 2, n. 1/2, p. 27-39, 2002.

TUCCI, C. A. F. Produção de mudas de espécies florestais nativas e implantação de sistema silvipastoril em várzea no município de Autazes, AM. Relatório técnico científico - FAPEAM, Processo: 894-2003. 2005. 24p.

TUCCI, C. A. F. et al. Calagem e adubação para produção de mudas de mogno (Swietenia macrophylla King.). Cerne, Lavras, v. 13, n. 3, p. 299-307. jul./set. 2007

VIEIRA, A. H. et al. Efeito de diferentes substratos para produção de mudas de freijó-louro (Cordia alliodora. Ruiz \& Pav.). Oken. Boletim de Pesquisa EMBRAPA - Centro de pesquisa agroflorestal do Acre, n. 25, p. 12, 1998.

YAGI, R. et al. Organic matter fractions and soil fertility under the influence of liming, vermicompost and cattle manure. Scientia Agricola, Piracicaba, v. 60, n. 3. p. 549-557, jul./set. 2003. 